\title{
Design and technological methods of reducing of thermal stress in grinding process
}

\author{
Tatyana $\mathrm{N}$. Ivanova ${ }^{1,2, *}$ \\ ${ }^{1}$ Tchaikovsky Branch Perm National Research Polytechnic University, 617764, Lenin St., 73, \\ Tchaikovsky, Russia \\ ${ }^{2}$ Federal State Budgetary Institution of Center "Udmurt Federal Research Center of the Ural Branch \\ of the Russian Academy of Sciences", Institute of Mechanics, 426000, T. Baramzinoy St., 34, \\ Izhevsk, Russia
}

\begin{abstract}
When grinding wheel comes in contact with machined surface thermal coupling occurs between the detail, shavings and cooling lubrication fluid. Depending on machining conditions, heat passes into detail machined from 50 to $83 \%$ which results in steel defects. Using results of carried research of thermal fields, thermal processes in grinding operations may be controlled. Through these processes we control quality of upper layer. As the result of carried research the next data are obtained: functional dependence, which allows calculating of rate of heating and cooling for every part of detail to be machined according to machining mode, time and intensity of heating source; dependence of stress calculation on operating time and temperature of heating source while grinding process is carried
\end{abstract}

Practical implementation of recommendations for controlling of thermal mode in grinding encounters some objective troubles $[1-5]$. Particularly, considerable decreasing of cutting speed, depth and supply for reducing of temperature to some appropriate safe level leads to serious decreasing of production output. By increasing cutting speed, rotational speed of wheel increases, bulge height of grains into machining surface decreases. As a result heating source intensity decreases and required accuracy and quality parameters are provided. Under small cutting speeds and low bulge height of grains released heat is not enough for structural changes. By increasing speed of detail, burns don't occur on machining area in spite of increasing load applied to one abrasive grain. However, speed of detail is limited because of possibility of occurrence of vibration which causes undulation of abraded surface to emerge. Grinding depth is one of the main parameters, determining production capacity. Increasing of it under constant speed of detail inevitably leads to increasing of temperature of upper layer of machined surface By changing proportions of speed of detail and intensity of grinding operation it is possible to control temperature of surface of detail within certain limits. Improving physical-and-chemical properties of

* Corresponding author: rsg078829@mail.ru 
technological lubrication means for cooling and developing technologies of their employment allows providing the least deformation of details by decreasing total amount of heat reflected to machined part.

The temperature in machining area may be considerably reduced in case of providing thermal up transient in grinding process by means of periodical intermittent interruption due to construction of cutting part of the wheel. As researches [2-8] show, it can be thought that optimal parameter of cutting surface of the wheel is occurrence of surface occupied by lubrication grooves on $20-25 \%$ of total work surface of grinding wheel.

Researches $[1,2]$ show that decreasing temperature in intermittent grinding occurs due to more favorable conditions of supplying of cooling lubrication liquid to cutting zone, because it is captured by grooves and then is delivered to contact area.

As a result of research equation expressing of temperature $T$ of working surface dependence on time in the next formula:

$$
T=\frac{2 q \cdot a}{\lambda \cdot \sqrt{\pi} \cdot v_{d}} \cdot\left[\frac{v_{d} \sqrt{\tau}}{2 \sqrt{a}}\left(1-F\left(\frac{v_{d} \sqrt{\tau}}{2 \sqrt{a}}\right)\right)-\frac{\exp \left(-\frac{v_{d}^{2} \sqrt{\tau}}{4 a}\right)-1}{\sqrt{\pi}}\right],
$$

where $q$ - density of heat flow, $a$ - coefficient of thermal diffusivity of the detail, $\lambda$ coefficient of thermal conductivity of the detail, $v_{d}$ - speed of the detail, $\tau$ - time of contact.

Or it can be expressed in criterion sample by adding criterion Pekle $P_{e}=\frac{v_{d} \cdot l}{2 a}$, criterion Furie $F_{o}=\frac{a \cdot \tau}{l^{2}}$ and relative temperature $\theta=\frac{\pi \cdot \lambda \cdot v_{d} \cdot T}{2 q \cdot a}$

$$
\theta=\sqrt{P_{e}^{2} \cdot F_{o}} \cdot\left(1-F\left(\sqrt{P_{e}^{2} \cdot F_{o}}\right)\right)-\frac{1}{\sqrt{\pi}} \cdot \exp \left[\left(-P_{e}^{2} \cdot F_{o}\right)-1\right]
$$

Temperature change of the surface in cooling process can be expressed the following way

$$
\frac{T-T_{c}}{T_{o}-T_{c}}=\exp \left[\left(\frac{\alpha}{\lambda}\right)^{2} \cdot a \cdot \tau_{c}\right] \cdot\left[1-F\left(\frac{\alpha}{\lambda} \cdot \sqrt{a \cdot \tau_{c}}\right)\right]
$$

where $T_{o}$ - body temperature in the beginning of cooling process; $T_{c}$ - temperature of cooling liquid; $\alpha$ - heat transfer coefficient, $\tau_{c}$ - time of cooling process.

Solution of the equation (3) in criterion sample:

$$
\theta_{c}=\exp T_{i}^{2}\left[1-F\left(T_{i}\right)\right]
$$

where $T_{i}=\frac{\alpha \cdot \sqrt{\tau_{c}}}{\sqrt{\lambda \cdot c \cdot \gamma}}$ - Tychonov criterion, c - coefficient of heat capacity.

The results of temperature field calculation of equations (1 - 4) for flat grinding by means of tool with intermittent work surface and by ejecting lubrication fluid for cooling in front of cutting area are shown on fig.1. Consumption of the lubrication fluid $Q_{c}=0.35$ litres per second, outflow velocity $v_{c}=3.76 \mathrm{mps}$, steel grade 3415 (12XH3A - Russian 
State Standard $(\mathrm{GOST}))\left(a=0.06 \mathrm{sm}^{2} / \mathrm{s}, \lambda=7.2 \mathrm{~W} /(\mathrm{m} \cdot \mathrm{K})\right)$, wheel speed $v_{w}=20 \mathrm{mps}$, detail speed $v_{d}=1.5$ meters per minute, wheel radius $R_{w}=75 \mathrm{~mm}$, width of contact area $H=5 \mathrm{~mm}$.

It is evident that maximum temperature while grinding by tool with entire cutting face is being $290{ }^{\circ} \mathrm{C}$. The highest temperature on the depth $\mathrm{x}=1 \mathrm{~mm}$ does not increase more than by $50{ }^{\circ} \mathrm{C}$ (fig. 1 , a). When grinding by intermittent tool with ejecting lubrication fluid for cooling, maximum temperature decreases by $15-20 \%$.
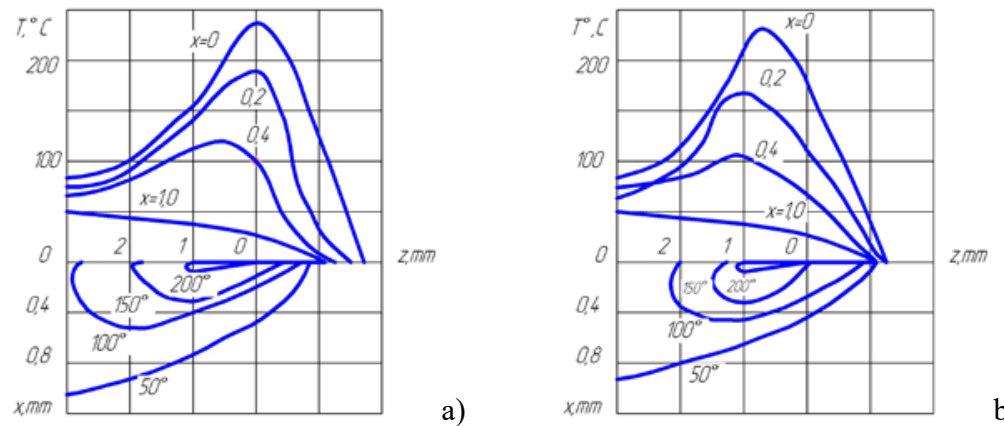

Fig. 1.Temperature profile of flat grinding of steel grade 3415 (12XH3A - Russian State Standard $($ GOST) ) a) tool with entire cutting face b) intermittent tool with ejecting lubrication fluid for cooling, Z-axis coordinated in points of contact, $\mathrm{x}$ - depth of thermal flow amplitude.

Moreover, changing of thermal field, representing temperature curves crossing for fixed depths, has occurred (fig. 1, b). Curve $\mathrm{x}=0$, corresponding to surface temperature, crosses curves, which represent the temperature of deeper layers. It means that after passing the source, temperature of deeper layers turns out to be greater than surface temperature. When $\mathrm{x}=0$ thermal gradient changes its direction after passing the source and some part of heat flow goes in upward direction to lubrication fluid for cooling. Temperature curves of deeper layers slightly deform. If heat transfer is present, penetration depth of high temperatures in grinding by means of intermittent tool decreases. Analysis of isotherms (bottom side of the graph fig.1) proves that part of heat flow goes deep inside the detail and causes its heating-up, and as for other part, it goes to surface of the detail and then inside the cooling agent. That is why the detail surface heats up to high temperatures under the source. Using of cooling considerably decreases penetration depth of high temperatures and total heat content of grinded detail.

In case of carrying out similar calculation for the source with bigger half-width of the contact $H$ influence of ejected fluid for cooling on the temperature will decrease. As for influence of flow of cooling lubricant, which is ejected directly in cutting area on heat transfer, it will grow. Decreasing of influence of ejected lubricant for cooling in case of bigger half-width of the contact $H$ is explained by the fact that heat transfer coefficient of the cooling lubrication fluid is constant, whereas heat transfer coefficient for flow of cooling lubricant can be changed by means of its thermophysical properties and quantity of grooves in the tool.

Dependence of heating depth $Z$ on half-width of the source $H$ is shown on fig. 2 . Increasing $H$ causes temperature to rise, maximum value of which shifts in opposite direction to source movement. When detail speed $v_{\mathrm{d}}$ reduces, decreasing of temperature and shifting of maximum point to the middle of the source. After passing the source, heating rate tends to zero and then changes sign - surface cooling occurs, more remote areas from surface keeping on heating. Time difference between cooling and heating of lower layers decreases by reducing detail speed. 
Being under the effect of cooling lubrication fluid and under similar properties and speed of heating source, maximum heating depth of surface layer $Z$ and temperature decrease (fig. 3).

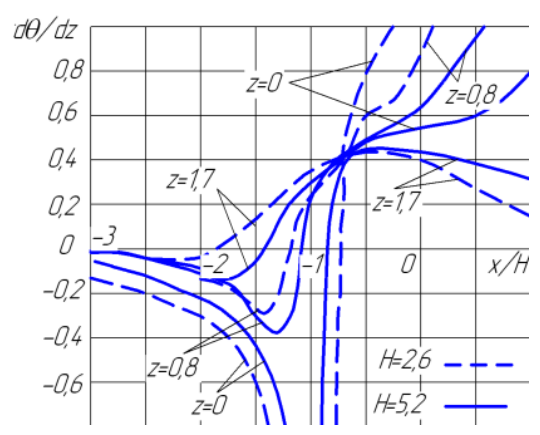

Fig. 2. Heating depth dependence on dimensionless quantity of half-width of the source

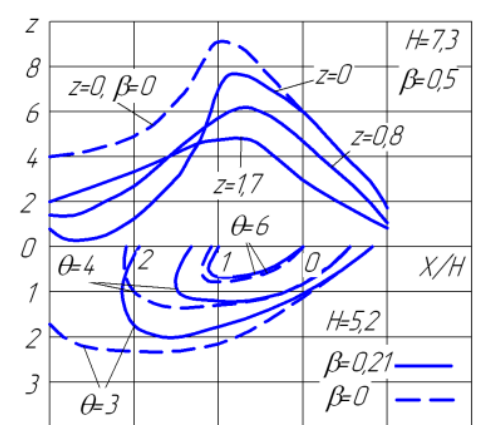

Fig. 3. Dependence of dimensionless temperature on warming-up of surface layer in case of heat transfer with cooling agent, $\beta$ - heat transfer coefficient.

According to obtained functional dependencies (fig. 1 - 3), speed of temperature changing has peak value if there is maximum detail movement, i.e. when operation time of the source $\tau$ is minimum value and consequently great thermal stresses in surface layer can be expected. By increasing $\tau$ thermal gradient reduces and stresses are likely to decrease. Under further increasing of $\tau$ the higher temperature influences machined detail, surface layers are heated deeper, as a result, there should be favorable conditions for the process of stress relaxation.

Further research allowed obtaining functional dependence of stress calculation on operation time and temperature of heating source in flat grinding:

$$
\sigma=\left(1-\frac{\sqrt{\pi \alpha \tau}}{2 z}\right) \cdot \alpha_{k} \operatorname{ET}(0, \tau)
$$

where $z$ - width of heated body, $T(0, \tau)$ - appropriate temperature on the surface and in $i$ layer, $E$ - elasticity modulus, $\alpha_{\kappa}$ - linear expansion coefficient of machined material.

Obtained functional dependence (5) shows that with decreasing heat time there is a tendency for stresses to increase. On the contrary, absolute value of stresses decreases by increasing heat time.

Equations (6) represent stress ratio in the moment of grinding and after grinding respectively:

$$
\sigma_{\theta_{c}}=\frac{\alpha \cdot E \cdot \theta_{F_{\max }} \cdot\left(1-e^{-\sqrt{\frac{x}{a \cdot t_{0}} \cdot x}}\right)}{2 \cdot(1-\mu)}, \sigma_{\theta_{s}}=\frac{\alpha \cdot E \cdot \theta_{\max (x)} \cdot\left(1-e^{-\sqrt{\frac{x}{a \cdot t_{0}} \cdot x}}\right)}{2 \cdot(1-\mu)}
$$

где $\alpha$ - linear expansion coefficient of material, $E$ - elasticity modulus, MPa, $\theta_{\text {Fmax }}$ temperature of surface layer of the, ${ }^{\circ} \mathrm{C}, \theta_{\max (x)}$ - temperature on fixed depth $\mathrm{Z},{ }^{\circ} \mathrm{C}, \mu$ poisson coefficient.

In the moment of grinding transient compression heat stresses occur on the surface, by further distancing from the surface they decrease and turn into tensile stress. After 
machining, when machined surface is being cooled, residual tensile stresses occur inside its surface layer. Experimental dependence of stresses on temperatures, occurring on the surface of the detail in grinding process is shown on fig. 4 .

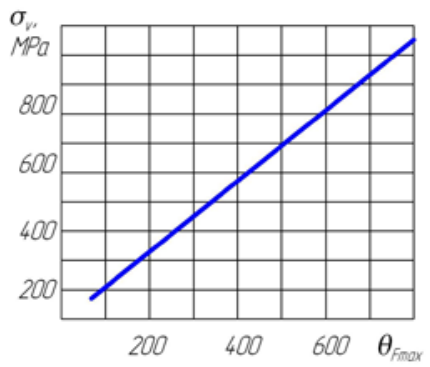

Fig. 4. Dependence of transient heat stresses on temperature

Theoretic and experimental researches of dependences 6 and analysis of fig. 4 show that crack formation in details made of steel grade steel grade 3415 (12XH3A - Russian State Standard (GOST)) occurs at temperatures higher than $650{ }^{\circ} \mathrm{C}$. In these conditions stresses reach $800 \div 1200 \mathrm{MPa}$, surpassing ultimate stress limit and causing fracturing in surface layers.

All in all, as the result of carried research into influence of operation time of heating source on machined detail the following was obtained:

- functional dependence, which allows calculating of rate of heating and cooling for every part of detail to be machined according to machining mode, time and intensity of heating source

- functional dependence of stress calculation on operating time and temperature of heating source when flat grinding process is carried

It was discovered that with decreasing heat time there is a tendency for stresses to increase, on the other hand, thermal gradient decreases by increasing heat time and the higher temperature influences the detail. It causes surface layer to heat deeper, that results into reducing of absolute value of stresses. Changing construction of cutting surface of grinding wheel and ratio of detail and wheel speeds as well as grinding depth, it is possible to control operation time of heat flow and reduce heat load density of flat grinding process.

\section{References}

1. F.Ju Svitkovskij, Choice of characteristics of diamond wheels in accordance with thermal mode of grain operation, Cutting and the tool issue 34 pp. 68-74 (1986)

2. T.N. Ivanova, A.M. Dolganov Up-to-date equipment in the technology of diamond face grinding of flat surfaces (Yekaterinburg - Izhevsk: the Institute of Economics Press, Ural Branch of RAS, 2007)

3. V.B. Dement'ev, T.N. Ivanova, A.M. Dolganov 2017 J Eng Phys Thermophy 90:102. doi:10.1007/s10891-017-1544-7

4. A.F. Rezchikov, A.V. Kochetkov, O.V. Zakharov. Mathematical models for estimating the degree of influence of major factors on performance and accuracy of coordinate measuring machines. MATEC Web Conf. Vol. 129. Pp. 01054 (2017)

5. D.I. Volkov Theoretical model of the stress state of the surface layer of components at deep grinding Solovyov Rybinsk State Aviation Technological Academy № 1 52-63 (2009).

6. G.V. Barsukov, L.G. Vainer, Yu.V. Vasilenko, A.M. Kozlov Modeling of technological processes of abrasive machining (Moscow: Spectr, 2011) 